NT@UW-01-06

LBNL 47692

RBRC-186

\title{
Towards a Perturbative Theory of Nuclear Forces
}

\author{
S.R. Beane ${ }^{a}$, P.F. Bedaque ${ }^{b}$, M.J. Savage ${ }^{a, c}$, and U. van Kolck B $^{d, e}$ \\ a Department of Physics, University of Washington, \\ Seattle, WA 98195 \\ ${ }^{b}$ Nuclear Science Division, Lawrence Berkeley National Laboratory, \\ Berkeley, CA 94720 \\ c Jefferson Laboratory, 12000 Jefferson Avenue, \\ Newport News, VA 23606 \\ ${ }^{d}$ Department of Physics, University of Arizona, \\ Tucson, AZ 85721 \\ e RIKEN-BNL Research Center, Brookhaven National Laboratory, \\ Upton, NY 11973
}

\begin{abstract}
We show that an expansion of nuclear forces about the chiral limit is formally consistent and is equivalent to KSW power counting in the ${ }^{1} S_{0}$ channel and Weinberg power counting in the ${ }^{3} S_{1}-{ }^{3} D_{1}$ coupled channels. Numerical evidence suggests that this expansion converges. The feasibility of making contact between nuclear physics and lattice-QCD simulations is discussed.
\end{abstract}




\section{Introduction}

The last decade has seen significant progress toward the formulation of an effective field theory (EFT) description of multi-nucleon systems (for recent reviews see Ref. [1, 2, 3, 4, 5]). The importance of uncovering such an EFT cannot be overstated as it is this theory alone that will allow for rigorous calculations of both elastic and inelastic processes in multi-nucleon systems in a framework consistent with the Standard Model of strong and electroweak interactions. An inherent advantage of an EFT framework is that the uncertainty associated with the computation of any given observable can be estimated and controlled with the power-counting scheme that defines the theory (along with the regulator). The fundamental difficulty in formulating power-counting schemes for multi-nucleon systems is that the interaction of two or more nucleons near threshold is intrinsically nonperturbative. This manifests itself through the presence of scattering lengths that are much larger than one would naively expect from $\mathrm{QCD}, \sim \Lambda_{Q C D}^{-1}$ [6]. For very low-momentum processes, such as $n p \rightarrow d \gamma$ in the nucleosynthesis region, a consistent and converging EFT, EFT( $九)$, has been developed to describe strong and electroweak processes in multi-nucleon systems [7]. However, attempts to develop a fully consistent theory to describe processes involving momenta larger than the mass of the pion have so far been unsuccessful.

Weinberg's (W) original proposal [6] for an EFT describing multi-nucleon systems was to determine the nucleon-nucleon (NN) potentials using the organizational principles of the well-established EFTs describing the meson-sector and single-nucleon sector (chiral perturbation theory), and then to insert these potentials into the Schrödinger equation to solve for NN wavefunctions. Observables are computed as matrix elements of operators (that have their own chiral expansion in this EFT) between these wavefunctions. W power counting has been extensively and successfully developed during the past decade to study processes in the few-nucleon systems (see, for example, Ref. [2]). This method requires numerical solution of the Schrödinger equation and is similar in spirit to traditional nuclear physics potential theory. Unfortunately, there are formal inconsistencies in $\mathrm{W}$ power counting [8], in particular, divergences that arise at leading order (LO) in the chiral expansion cannot be absorbed by the LO operators. Problems persist at all orders in the chiral expansion, and the correspondence between divergences and counterterms appears to be lost. This formal issue was partially solved by Kaplan, Savage and Wise (KSW) who introduced a power counting in which pions are treated perturbatively [9]. The NN phase-shifts and mixing angle in the ${ }^{1} S_{0}$ and ${ }^{3} S_{1}-{ }^{3} D_{1}$ channels have been computed at next-to-next-to-leading order $\left(\mathrm{N}^{2} \mathrm{LO}\right)$ in the KSW expansion by Fleming, Mehen and Stewart (FMS) [10 from which it can be concluded that the KSW expansion converges slowly in the ${ }^{1} S_{0}$ channel and does not converge at all in the ${ }^{3} S_{1}-{ }^{3} D_{1}$ coupled channels. FMS found that the part of the scattering amplitude that survives in the chiral limit is large enough to destroy convergence in the ${ }^{3} S_{1}-{ }^{3} D_{1}$ channels. This is in contrast with converging numerical results found using $W$ power counting in this channel [11], [12].

Both W and KSW power countings have desirable features that one would hope exist in the, yet to be constructed, EFT that describes multi-nucleon systems. It is therefore 
important to consider two fundamental questions in this area:

1. Where is $W$ power counting consistent? It has been argued that there are fundamental problems with the chiral expansion and the momentum expansion resulting from $\mathrm{W}$ power counting 8 . In this paper we confirm that $\mathrm{W}$ power counting is formally inconsistent in the ${ }^{1} S_{0}$ channel, but we do find that $\mathrm{W}$ power counting is consistent in the ${ }^{3} S_{1}-{ }^{3} D_{1}$ coupled channels. The arguments presented in Ref. [8] were based on a perturbative analysis, whereas the correct renormalization of singular potentials is intrinsically nonperturbative [13, 14, 15]. As we will see the same is true in the ${ }^{3} S_{1}-{ }^{3} D_{1}$ coupled channels.

2. Where is pion exchange perturbative? The analysis of FMS demonstrated that pions, or more explicitly, the Yukawa part of the NN potential and radiation pions, when treated perturbatively give rise to a converging expansion for the ${ }^{1} S_{0}$ scattering amplitude. They also showed that one-pion-exchange (OPE) in the ${ }^{3} S_{1}-{ }^{3} D_{1}$ coupled channels is not perturbatively convergent. In this paper we confirm the result of Ref. [8] that part of the pion contribution to scattering in the ${ }^{1} S_{0}$ channel requires perturbative treatment in order to obtain a consistent theory. However, there is no such requirement in the ${ }^{3} S_{1}-{ }^{3} D_{1}$ coupled channels.

In this paper we will argue, supported by numerical evidence, that an expansion about the chiral limit provides a systematic power counting for multi-nucleon systems. This expansion is found to be equivalent to KSW power counting in the ${ }^{1} S_{0}$ channel and equivalent to $\mathrm{W}$ power counting in the ${ }^{3} S_{1}-{ }^{3} D_{1}$ coupled channels, i.e. it selects only the desirable features of both power countings. To understand the physics behind this expansion, it is sufficient to recall that in the ${ }^{3} S_{1}-{ }^{3} D_{1}$ coupled channels, OPE includes a strong tensor component at short distances that persists in the chiral limit and is large enough so that it must be summed to all orders, as required by $\mathrm{W}$ power counting. However, deviations from the chiral limit $V\left(r ; m_{\pi}\right)-V(r ; 0)$ can be treated perturbatively in all channels, and in fact, such a perturbative expansion is required in the ${ }^{1} S_{0}$ channel but not in the ${ }^{3} S_{1}-{ }^{3} D_{1}$ channel.

In section 2 we discuss the ${ }^{1} S_{0}$ channel. In particular, we review the problems with W power counting in this channel. Using the Standard Toy Model of Nuclear Physics (the three-Yukawa model developed in Ref. [16]), detailed in the Appendix, we show that the Yukawa part of OPE can be treated perturbatively if the short-distance physics is properly accounted for in the EFT. We outline a method for improving the convergence of the ${ }^{1} S_{0}$ phase-shift in the realistic case. In section 3 we investigate the ${ }^{3} S_{1}-{ }^{3} D_{1}$ coupled channels. The LO amplitude in $\mathrm{W}$ power counting is analytically renormalized and a comparison with numerical data is presented. The NN phase-shifts are computed at high orders in perturbation theory to study the convergence of the $V\left(r ; m_{\pi}\right)-V(r ; 0)$ expansion. In section 4 we discuss possible scenarios for the light-quark mass dependence of the deuteron binding energy and NN scattering amplitudes, and dimly illuminate the long and winding road between nuclear physics and lattice QCD. We conclude in section 5 . 


\section{Scattering in the ${ }^{1} S_{0}$-Channel}

NN scattering in the ${ }^{1} S_{0}$ channel has been studied extensively in EFT investigations as it results from a relatively simple interaction between nucleons. OPE provides the longdistance Yukawa interaction and there is a large short-distance interaction, affectionately known as the hard-core. This structure makes the ${ }^{1} S_{0}$ channel a useful testing ground for new ideas.

The ${ }^{1} S_{0}$ channel has been investigated using both W and KSW power countings with interesting results. First, calculations up to $\mathrm{N}^{2} \mathrm{LO}$ in $\mathrm{W}$ power counting have been performed [11, 12] and reproduce the phase-shift data in this channel and in many other channels very well for a range of ultra-violet (UV) momentum cut offs. However, there is a sensitivity to the momentum cut off due to an inconsistency in $\mathrm{W}$ power counting [8]. Second, calculations up to $\mathrm{N}^{2} \mathrm{LO}$ in KSW power counting have been performed [9, 10], and are found to converge to the results of the Nijmegen phase-shift analysis [17]. The convergence of the expansion can be improved substantially by promoting the effective range from NLO to LO in KSW power counting [16], implemented via the dibaryon field [18, 19] or the z-parameterization [20].

\subsection{Problems with W Power Counting in the ${ }^{1} S_{0}$ Channel}

In $\mathrm{W}$ power counting the momentum-independent four-nucleon interaction and $\mathrm{OPE}$ form the LO NN interaction. The Schrödinger equation is used to determine the LO phaseshifts for NN scattering from this LO interaction. This procedure corresponds to summing an infinite number of insertions of the LO interaction. Naively, this would appear to be a sensible procedure, however, subtle problems arise in solving the Schrödinger equation due to its bad UV-behavior and it must be renormalized, as discussed in detail in Ref. [8].

The OPE central and tensor potentials are

$$
V_{C}\left(r ; m_{\pi}\right)=-\alpha_{\pi} m_{\pi}^{2} \frac{e^{-m_{\pi} r}}{r}
$$

and

$$
V_{T}\left(r ; m_{\pi}\right)=-\alpha_{\pi} m_{\pi}^{2} \frac{e^{-m_{\pi} r}}{r}\left(1+\frac{3}{m_{\pi} r}+\frac{3}{m_{\pi}^{2} r^{2}}\right),
$$

respectively, where

$$
\alpha_{\pi}=\frac{g_{A}^{2}}{16 \pi F_{\pi}^{2}}
$$

and we use $g_{A}=1.26, F_{\pi}=93 \mathrm{MeV}$ and $M=940 \mathrm{MeV}$. Scattering in the ${ }^{1} S_{0}$ channel depends only on the central potential, $V_{C}$. Near the origin, $V_{C}$ is Coulombic 1 and gives rise to NN wavefunctions that are a linear combination of regular and irregular Bessel functions. At LO in $\mathrm{W}$ power counting, OPE is renormalized by a single delta-function

\footnotetext{
${ }^{1}$ The $\delta^{(3)}(r)$ contribution to $V_{C}$ is not shown explicitly but is absorbed into the definition of the local four-nucleon operator defined in eq. 4 .
} 
counterterm, $C_{0}$. For the purposes of this paper, we choose to smear the delta-function counterterm(s) over a finite volume near the origin and for computational ease choose a square well [21] of radius $R$,

$$
C_{0} \delta^{(3)}(r) \rightarrow \frac{3 C_{0} \theta(R-r)}{4 \pi R^{3}} \equiv V_{0} \theta(R-r) .
$$

The OPE potential is similarly cutoff at the radius $R \equiv 1 / \Lambda$. The advantage of this simple regulator is that analytic matching can be performed f], and at zero center-of-mass (CoM) energy one finds

$$
\sqrt{-M V_{0}} \cot \left(\sqrt{-M V_{0}} R\right)=-m_{\pi}^{2} M \alpha_{\pi} \log \left(\frac{R}{R_{*}}\right)+\mathcal{O}(R)
$$

where $R_{*}$ is an intrinsic length scale to be determined numerically. Treating $\sqrt{-M V_{0}} R$ as an expansion in $m_{\pi}$, we find the depth of the energy-independent square well as a function of $R$ to be

$$
V_{0}(R ; n)=-(2 n+1)^{2} \frac{\pi^{2}}{4 M R^{2}}-\frac{2 m_{\pi}^{2} \alpha_{\pi}}{R} \log \left(\frac{R}{R_{*}}\right)+\mathcal{O}\left(R^{0}\right) .
$$

where $n$ labels the branch of the cotangent. The presence of a multi-branch structure is

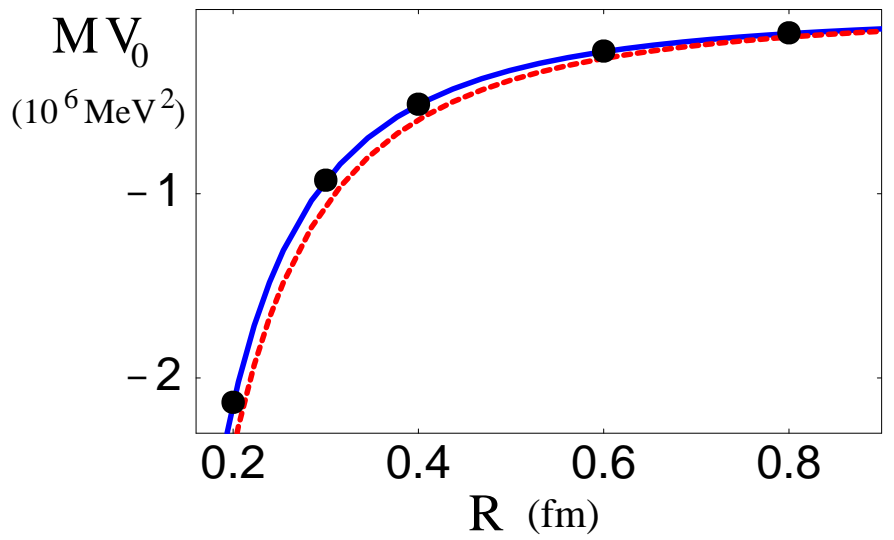

Figure 1: The solid line represents the running of $V_{0}$ as a function of the cutoff $R$ (in fermis), taken from eq. (6) with $n=1$, for the physical value of $m_{\pi}$, while the dotted line neglects the $R^{-1}$ part of the running. The dots are extracted directly from a numerical solution of the Schrödinger equation for the physical value of $m_{\pi}$.

related to the accumulation of bound states inside the square well. Of course the presence of unphysical bound states is innocuous as long as the binding energies of such states

\footnotetext{
${ }^{2}$ The physics we obtain will be regulator independent. Nevertheless, we have been unable to dimensionally regulate the ${ }^{3} S_{1}-{ }^{3} D_{1}$ coupled channels.
} 
are near the cutoff of the EFT. In fig. 1 the $R$ dependence of $V_{0}$, as given by eq. (6), is compared to points extracted from the numerical solution of the Schrödinger equation. For a given $R, V_{0}$ is tuned to give the scattering length, $a^{1} S_{0}=-23.714 \mathrm{fm}$, and in fig. 2 we show the ${ }^{1} S_{0}$ phase-shifts for different values of $R$. In momentum-space notation the matching equation takes the form:

$$
C_{0}(\Lambda ; n)+m_{\pi}^{2} D_{2}(\Lambda)=(2 n+1)^{2} \frac{\pi^{3}}{3 M \Lambda}+\frac{8 \pi m_{\pi}^{2} \alpha_{\pi}}{3 \Lambda^{2}} \log \left(\frac{\Lambda_{*}}{\Lambda}\right),
$$

and therefore the $D_{2}$ operator, which is formally subleading in $\mathrm{W}$ power counting, must be promoted to $L O$, in agreement with the perturbative argument of Ref. [®]. Although the logarithmic divergence is suppressed by a power of $\Lambda$ compared to the leading term in eq. 7, it is a true divergence in physical quantities which must be renormalized at leading order in $\mathrm{W}$ power counting (see eq. 10 below). This offers a proof that in the ${ }^{1} S_{0}$ channel, an expansion of the action to a given order in $\mathrm{W}$ power counting does not consistently remove all cutoff dependence to that order [22]. Of course the $D_{2}$ contribution is numerically small, as demonstrated by the dotted curve in fig. 11 which neglects the $\Lambda^{-2}$ corrections to the running. This smallness explains why W power counting has been found to work well in this channel over a moderate range of cut offs [11, 22, 12, 3].
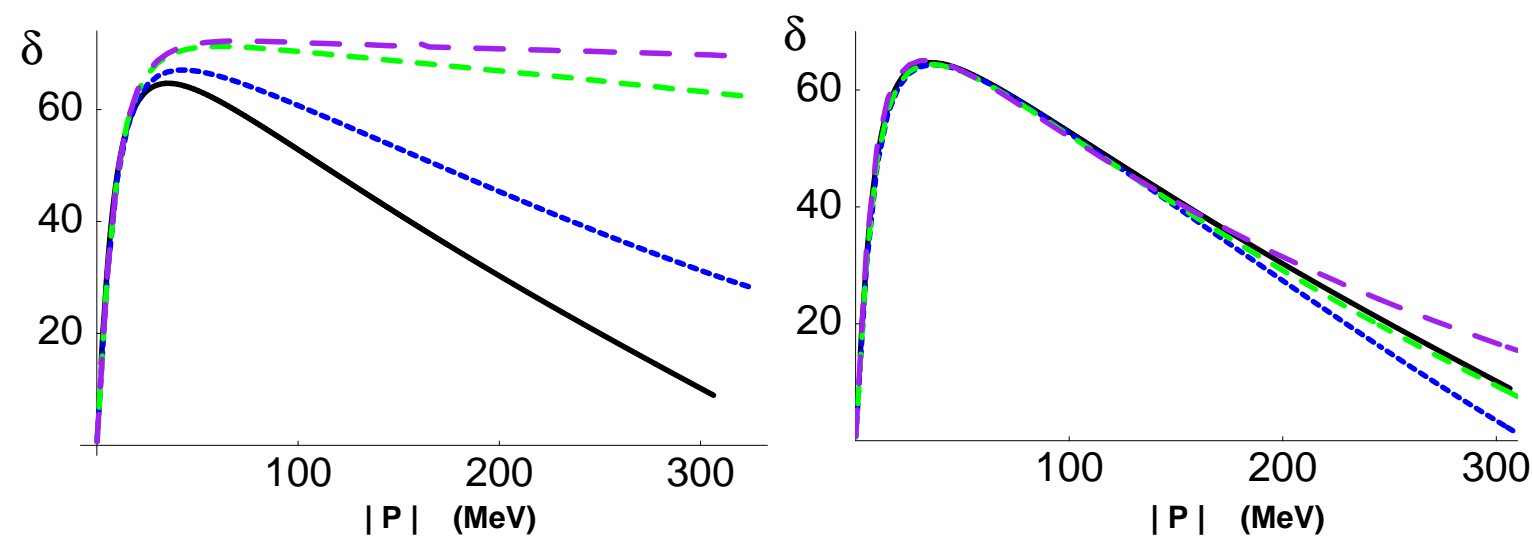

Figure 2: The ${ }^{1} S_{0}$ phase-shift plotted versus CoM momentum. The solid lines are the Nijmegen phase-shift analysis [17]. The long-dash lines corresponds to $R=0.2 \mathrm{fm}(\Lambda=$ $985 \mathrm{MeV})$, the medium-dash lines correspond to $R=0.4 \mathrm{fm}(\Lambda=492 \mathrm{MeV})$, and the dotted lines correspond to $R=1.4 \mathrm{fm}(\Lambda=140 \mathrm{MeV})$. The left panel corresponds to setting $V_{2}=0$, while the right panel includes $V_{2}$ in such a way to reproduce the measured effective range, $r^{1} S_{0}$.

How does this problem manifest itself at the level of Feynman diagrams [8]? In dimensional regularization the divergent part of diagram (a) in fig. 3 is

$$
-\frac{1}{\epsilon} \frac{g_{A}^{2} m_{\pi}^{2} M^{2}}{256 \pi^{2} F_{\pi}^{2}} C^{2}
$$


where $C$ is the coefficient of the four-nucleon operator, and $n=4-2 \epsilon$ is the number of space-time dimensions. This diagram requires a counterterm, $D_{2}$, with a single insertion of the light quark mass matrix, $m_{q}$, to render the amplitude finite, however, such counterterms occur only at higher orders in the chiral expansion. Thus, the inconsistency of $\mathrm{W}$ power counting is unmasked diagrammatically.

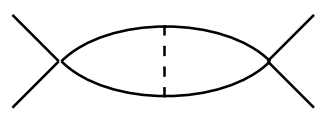

(a)

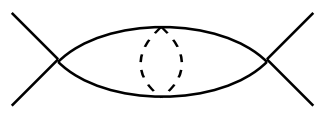

(b)

Figure 3: Graphs with logarithmic divergences at (a) LO in $W$ power counting and NLO in KSW counting and (b) NLO in W power counting and $N^{2} L O$ in KSW counting. The solid lines are nucleons and the dashed lines are pions.

It is instructive to understand how the divergence in eq. (8) arises in position space with a square-well regulator. The radial wavefunctions resulting from resumming the LO bubbles are phase-shifted plane-waves of the form

$$
u(r) \sim \sin \left[k r+\delta_{\mathrm{LO}}\right],
$$

where $\delta_{\mathrm{LO}}$ is the LO phase-shift. Perturbatively inserting the difference between the OPE potential for $m_{q} \neq 0$ and its value in the chiral limit, and regulating the UV divergence with a square well of a radius $R$ much smaller than the scattering length $R \ll a$, the NLO contribution to the scattering amplitude is

$$
\begin{aligned}
\left\langle\psi^{\prime}\left|V\left(m_{\pi}\right)-V(0)\right| \psi\right\rangle & \sim g_{A}^{2} m_{\pi}^{2} \int_{R}^{\infty} d r \sin ^{2}\left[k r+\delta_{\mathrm{LO}}\right] \frac{\exp \left(-m_{\pi} r\right)}{r} \\
& \sim-g_{A}^{2} m_{\pi}^{2} \sin ^{2} \delta_{\mathrm{LO}} \log \left(m_{\pi} R\right)+\ldots
\end{aligned}
$$

where the ellipses denote terms that are finite as $R \rightarrow 0$. At the order at which $V\left(m_{\pi}\right)-$ $V(0)$ enters, there must be a contribution from a local operator of the form $m_{\pi}^{2} \log \left(R / R_{*}\right)$, where $R_{*}$ is some intrinsic length scale, in order to render the sum finite 3 and independent of the renormalization scale $R$.

By the analysis above, one is naturally led to the conclusion that the contribution from OPE and the $\mathcal{O}\left(m_{q}\right)$ counterterms must contribute to any amplitude at the same order in the expansion, and this is what leads to KSW power counting. However, a more general conclusion to draw is that the difference between the OPE contribution for $m_{q} \neq 0$ and the OPE contribution in the chiral limit must occur at the same order as the $\mathcal{O}\left(m_{q}\right)$ counterterms. In most cases, these two conclusions yield identical amplitudes, however, in the ${ }^{3} S_{1}-{ }^{3} D_{1}$ channel they do not, as we will see subsequently.

\footnotetext{
${ }^{3}$ If the LO radial wavefunctions behaved differently as $r \rightarrow 0$, the divergence structure would be modified. Such a modification can only arise from a re-ordering of the perturbative expansion, and consequently the hierarchy of higher-dimension operators as determined from naive arguments will also be modified.
} 


\subsection{Are Pions Perturbative?}

In KSW power counting the renormalization group (RG) flow of the LO four-nucleon interaction promotes it to one lower order in the chiral expansion, while the OPE interaction does not evolve. The LO NN scattering amplitude arises from resumming the bubbles with an infinite number of insertions of the four-nucleon interaction. At NLO, there are contributions from OPE, insertions of $\mathcal{O}\left(m_{q}\right)$ four-nucleon operators and from insertions of $\mathcal{O}\left(|\mathbf{p}|^{2}\right)$ operators. Operationally, this hierarchy arises from the assignment that the scattering length scales as an inverse power of the small expansion parameter, $a \sim 1 / Q$, while all other parameters are natural size. The scattering amplitude in the ${ }^{1} S_{0}$ channel has been computed to $\mathrm{N}^{2} \mathrm{LO}$ in KSW power counting in Ref. [10], where it was found to converge slowly. However, the cause of the slow convergence with KSW in this channel stems from the treatment of the short-distance contribution to the scattering amplitude and not from perturbative OPE. All momentum dependence arising from the hard-core of the NN interaction is treated in perturbation theory and as these effects are large, even over a relatively small momentum range, the convergence is slow.

\subsection{Lessons from a Model}

A toy model that has proved useful in understanding the structure of the NN interaction is the three-Yukawa model f. One includes a Yukawa pion, rho and sigma meson, with their physical masses (the sigma meson is given a mass of $550 \mathrm{MeV}$, despite its non-existence in nature) and their couplings are adjusted to reproduce the scattering length and effective range in the ${ }^{1} S_{0}$ channel. Convergence problems with KSW power counting were highlighted with this model by Steele and Kaplan [16], and it is important to understand the cause of these problems before proceeding further.

Several valuable lessons about both KSW and W power counting formulations can be learned from the STMoNP. First, pions are perturbative in the ${ }^{1} S_{0}$ channel. The full theory defined via the Schrödinger equation can be systematically perturbed in the number of insertions of the pion potential. In addition, one can recover these perturbative amplitudes with an EFT construction provided one retains a sufficient number of counterterms. Second, the fact that W power counting 1 converges nicely, while KSW converges quite slowly [16], and appears to encounter problems at a scale $\sim m_{\pi}$ is now understood. The problem is not with the perturbative pions component of KSW but rather with the treatment of the short-distance physics. By resumming the "hard-core" into a dibaryon field, this modified-KSW (or modified-W) power counting does provide a perturbativelyconvergent amplitude. The peculiar feature, however, is that by only including a small number of local counterterms and by eyeballing the phase-shift plots, one would guess that the breakdown scale for the theory is $\sim m_{\pi}$. However, a more systematic analysis,

\footnotetext{
${ }^{4}$ We will refer to this model, which we detail in the Appendix, as the Standard Toy Model of Nuclear Physics (STMoNP).

${ }^{5}$ In the STMoNP, W power counting does not lead to any ambiguities because the long-distance component of the nucleon interaction is sufficiently UV convergent.
} 
such as that provided by error plots or by going to higher orders in the expansion, reveals a breakdown scale of $m_{\sigma} / 2$, set by the radius of convergence of the effective range expansion for the short-distance physics. Third, it is clear from this analysis that $\mathrm{W}$ power counting is overkill. One does not need to resum pions exchanges in order to arrive at a meaningful phase-shift for this theory, and in fact, closed-form analytic expressions exist and reproduce the numerical solutions of the Schrödinger equation up to $m_{\sigma} / 2$.

Note that the agreement with data (in the left panel of fig. 2) breaks down at very-low momenta because the scattering length is large and the effective range in this channel is large as well, $r^{1} S_{0}=2.73 \mathrm{fm}$, comparable to twice the pion Compton wavelength. This suggests that the two-derivative $C_{2}$ operator should be promoted to LO, and is easily included in our analysis via an energy-dependent contribution to the depth of the square well:

$$
C_{2} \nabla^{2} \delta^{(3)}(r) \rightarrow C_{2} M \bar{E} \delta^{(3)}(r) \rightarrow \frac{3 C_{2} k^{2} \theta(R-r)}{4 \pi R^{3}} \equiv k^{2} V_{2} \theta(R-r)
$$

where $\bar{E}=k^{2} / M$ is the CoM energy. The first step amounts to a field redefinition which removes momentum dependence in favor of energy dependence [19, 23, 24]. The value of $V_{2}$ as a function of $R$, is chosen to recover the experimental value of $r^{1 S_{0}}$. In the right panel of fig. 2 we see the much-improved phase-shift with resummed range corrections, providing an example of the manner in which the momentum expansion can be tailored to experimental realities 19 .

\section{Scattering in the ${ }^{3} S_{1}-{ }^{3} D_{1}$ Coupled Channels}

In addition to the long-distance Yukawa OPE interaction and the hard-core interaction, NN scattering in the ${ }^{3} S_{1}-{ }^{3} D_{1}$ coupled channels has a strong tensor component. At distances $r \ll m_{\pi}$ the central potential is negligible and the nucleons interact entirely through the singular tensor interaction. The chiral expansion of the tensor force is equivalent to the short-distance expansion and is given by

$$
V_{T}\left(r ; m_{\pi}\right)=-\frac{3 \alpha_{\pi}}{r^{3}}+\frac{m_{\pi}^{2} \alpha_{\pi}}{2 r}+\mathcal{O}\left(m_{\pi}^{4}\right)
$$

It is the leading term in this expansion which leads to the breakdown of the KSW expansion in the ${ }^{3} S_{1}-{ }^{3} D_{1}$ coupled channels [10]. Note that a significant consequence of chiral symmetry is that there is no $1 / r^{2}$ term in the short-distance expansion of $V_{T}\left(r ; m_{\pi}\right)$. One may hope that the convergence problems in the ${ }^{3} S_{1}-{ }^{3} D_{1}$ coupled channels can be cured as in the ${ }^{1} S_{0}$ channel by a re-ordering of local operators representing this short distance physics. Unfortunately, this is not the case. For potentials of the form $1 / r^{n}$, with $n \leq 3$, $k \cot \delta$ is non-analytic in $k$ (for a comprehensive review of singular potentials see Ref. [14]) and consequently the chiral limit of $V_{T}\left(r ; m_{\pi}\right)$ cannot be represented by local operators, and must be summed to all orders. At first glance, this might seem to pose an insurmountable problem for the EFT effort. KSW have shown that if one isolates terms in 
perturbation theory with insertions of the tensor force, it appears that an infinite number of counterterms are necessary to properly renormalize the highly-singular behavior. This was motivation for treating the pion perturbatively. Fortunately, this argument is not correct, essentially because it relies on perturbation theory, and the correct renormalization is invisible in perturbation theory. This is similar to what occurs in the three-body system with short-ranged interactions [25]. In recent work, some of us showed that a $1 / r^{n}$ attractive singular potential can be renormalized by a single energy-independent square well [15]. Here we will extend this result to the realistic case, which amounts to showing that the momentum expansion assumed by $\mathrm{W}$ power counting is consistent in the ${ }^{3} S_{1}-{ }^{3} D_{1}$ coupled channels. The investigation is in similar spirit to the work in Ref. [26] who consider an OPE potential and work with a subtracted T-matrix, and to the work in Ref. [27] which examines scattering and electromagnetic observables in the ${ }^{3} S_{1}-{ }^{3} D_{1}$ coupled channels.

\subsection{W Power Counting at LO}

At LO in $\mathrm{W}$ power counting, OPE in the ${ }^{3} S_{1}-{ }^{3} D_{1}$ coupled channels is renormalized by a single delta-function counterterm, as it is in the ${ }^{1} S_{0}$-channel. For demonstrative purposes, we again choose to regulate the delta-function with a square well, which has been implemented previously in very forward-thinking work by Sprung et al [28]. The $S$-wave and $D$-wave components of the $J=1$ system, $u(r)$ and $w(r)$ respectively, are coupled by the tensor potential, $V_{T}$, defined in eq. (2). The potential outside the square well is

$$
\mathcal{V}_{L}\left(r ; m_{\pi}\right)=\left(\begin{array}{cc}
-M V_{C}\left(r ; m_{\pi}\right) & -2 \sqrt{2} M V_{T}\left(r ; m_{\pi}\right) \\
-2 \sqrt{2} M V_{T}\left(r ; m_{\pi}\right) & -M\left(V_{C}\left(r ; m_{\pi}\right)-2 V_{T}\left(r ; m_{\pi}\right)\right)-6 / r^{2}
\end{array}\right),
$$

while the potential inside the square well is

$$
\mathcal{V}_{S}(r)=\left(\begin{array}{cc}
-M\left(V_{0}+k^{2} V_{2}\right) & 0 \\
0 & -M\left(V_{0}+k^{2} V_{2}\right)-6 / r^{2}
\end{array}\right)
$$

Here we have included $V_{2}$, but of course it is not necessary at LO in $\mathrm{W}$ counting. We have also left out ${ }^{3} S_{1}-{ }^{3} D_{1}$ superscripts which differentiate this square well from that which appears in the ${ }^{1} S_{0}$ channel. Defining $\Psi$ to be

$$
\Psi(r)=\left(\begin{array}{c}
u(x) \\
w(x)
\end{array}\right),
$$

the regulated Schrödinger equation is

$$
\Psi^{\prime \prime}(r)+\left(k^{2}+\mathcal{V}_{L}\left(r ; m_{\pi}\right) \theta(r-R)+\mathcal{V}_{S}(r) \theta(R-r)\right) \Psi=0
$$

Renormalization concerns the behavior of physics at short-distances, or more specifically, is due to our lack of understanding of physics at short-distances. If $R<r \ll M \alpha_{\pi} \equiv$ $1 / \Lambda_{N N}$, then we can neglect the angular-momentum barrier and keep only the chiral 
limit of the tensor force. Moreover, for $|k| \ll \Lambda_{N N}$ the total energy can be treated as a perturbation. In this short-distance limit, $\mathcal{V}_{L}\left(r ; m_{\pi}\right)$ can be diagonalized and the Schrödinger equation can be solved exactly. In the diagonal basis the Schrödinger equation decouples into an attractive singular potential and a repulsive potential. The solution for the attractive singular potential, $\bar{u}$, is a linear combination of Bessel functions [28, 15], and it is useful to think of the short-distance limit of the Bessel functions using the WKB approximation [13]. The first two orders in the WKB approximation reproduce the LO asymptotic expansion of the Bessel functions, while NLO in WKB gives the leading energy corrections. The wavefunction at this order is

$$
\bar{u}(r ; k)=A r^{3 / 4} \cos \left(2 \sqrt{\frac{6 \alpha_{\pi} M}{r}}-\frac{r^{2} k^{2}}{5} \sqrt{\frac{r}{6 \alpha_{\pi} M}}+\phi_{0}+k^{2} \phi_{1}\right),
$$

where $A$ is a dimensionful normalization constant and $\phi_{0}$ and $\phi_{1}$ are the coefficients of a Taylor-series expansion of the asymptotic phase $\phi\left(k^{2}\right)$. Note that this wavefunction vanishes at the origin.

The solution for $r<R$ is simply that of a square well of height $V_{0}+k^{2} V_{2}$. Matching logarithmic derivatives of the interior square well and exterior attractive solutions at $r=R$ yields the matching equation

$$
\sqrt{-M V_{0}} R \cot \left(\sqrt{-M V_{0}} R\right)=\frac{3}{4}+\sqrt{\frac{6 M \alpha_{\pi}}{R}} \tan \left(2 \sqrt{\frac{6 M \alpha_{\pi}}{R}}+\phi_{0}\right)
$$

for $V_{0}$ and

$$
\begin{gathered}
\frac{\left(M V_{2}-1\right)}{M V_{0}}\left[\sqrt{-M V_{0}} \cot \left(\sqrt{-M V_{0}} R\right)+M V_{0} R \csc ^{2}\left(\sqrt{-M V_{0}} R\right)\right]= \\
\frac{R^{3 / 2}}{\sqrt{6 M \alpha_{\pi}}} \tan \left(2 \sqrt{\frac{6 M \alpha_{\pi}}{R}}+\phi_{0}\right)-\left(\frac{2}{5} R+\frac{2}{R} \sqrt{\frac{6 M \alpha_{\pi}}{R}} \phi_{1}\right) \sec ^{2}\left(2 \sqrt{\frac{6 M \alpha_{\pi}}{R}}+\phi_{0}\right)
\end{gathered}
$$

for $V_{2}$. These equations determine the renormalization-group flow of $V_{0}$ and $V_{2}$. Note that this flow is multibranched and non-analytic in the coupling $\alpha_{\pi}$ ? The phases $\phi_{0}$ and $\phi_{1}$ are determined numerically. For a given $R, V_{0}$ is tuned to recover the physical value of the scattering length in the ${ }^{3} S_{1}$ channel, $a^{\left({ }^{3} S_{1}\right)}=5.425 \mathrm{fm}$, from which eq. (18) determines the phase $\phi_{0}$. $V_{2}$ is chosen to recover the correct physical value of the effective range, $r^{\left({ }^{3} S_{1}\right)}=1.749 \mathrm{fm}$ from which eq. (19) determines $\phi_{1}$. In fig. the square-well depths $V_{0}$ and $V_{2}$ are shown as functions of the square-well width $R$ taken from eq. (18) and eq. (19). The LO phase-shifts in the ${ }^{3} S_{1}-{ }^{3} D_{1}$ coupled channels are shown in fig. 5 and fig. 6 with $V_{2}=0$. While the phase-shifts $\delta_{0}$ and $\delta_{2}$ are clearly independent of the square-well radius $R$, the mixing parameter $\varepsilon_{1}$ exhibits $R$ dependence. However, an error plot of $\varepsilon_{1}$ indicates that the $R$ dependence and the deviations from the Nijmegen phase-shift analysis are higher order in the momentum expansion, beginning at $\mathcal{O}\left(k^{2}\right)$ (and do not indicate an inconsistency in the power counting). 

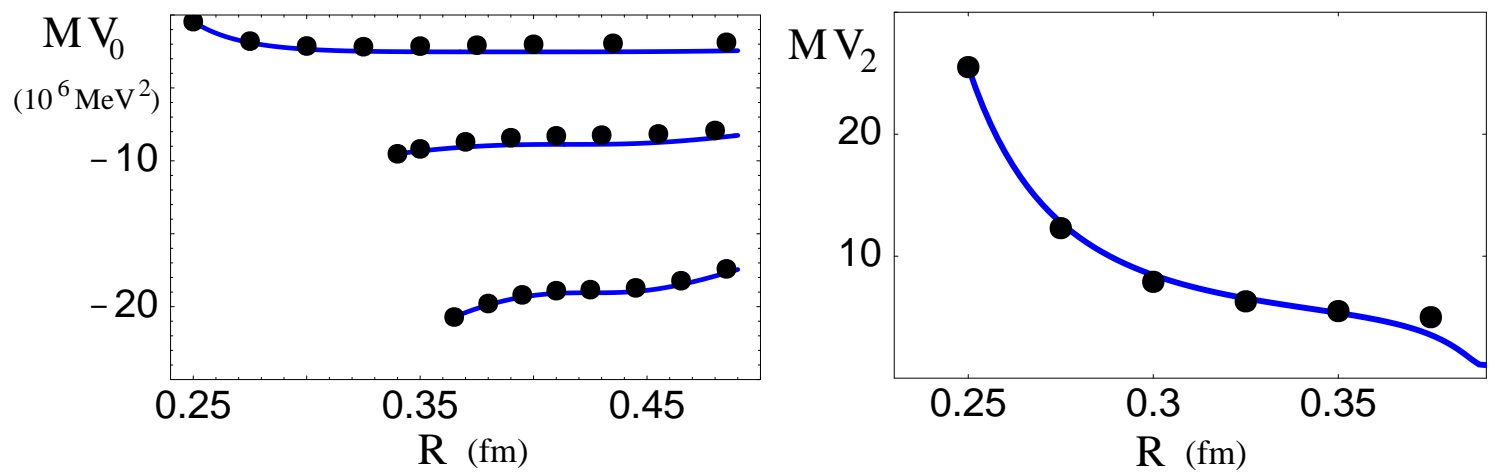

Figure 4: The solid lines represent the running of $M V_{0}$ and $M V_{2}$ as a function of the cutoff $R$, taken from eq. (18) and eq. (19), respectively. The dots are extracted directly from a numerical solution of the Schrödinger equation. The different branches in the left panel correspond to a different number of nodes in the square well, i.e. an artifact of this particular regulator. The curves continue to smaller values of $R$, but we have not shown them. Further, analogous branches exist for $M V_{2}$, but we have not shown them. For each branch, the ultraviolet phase is fit to the smallest $R$ data point to produce the theoretical curve.

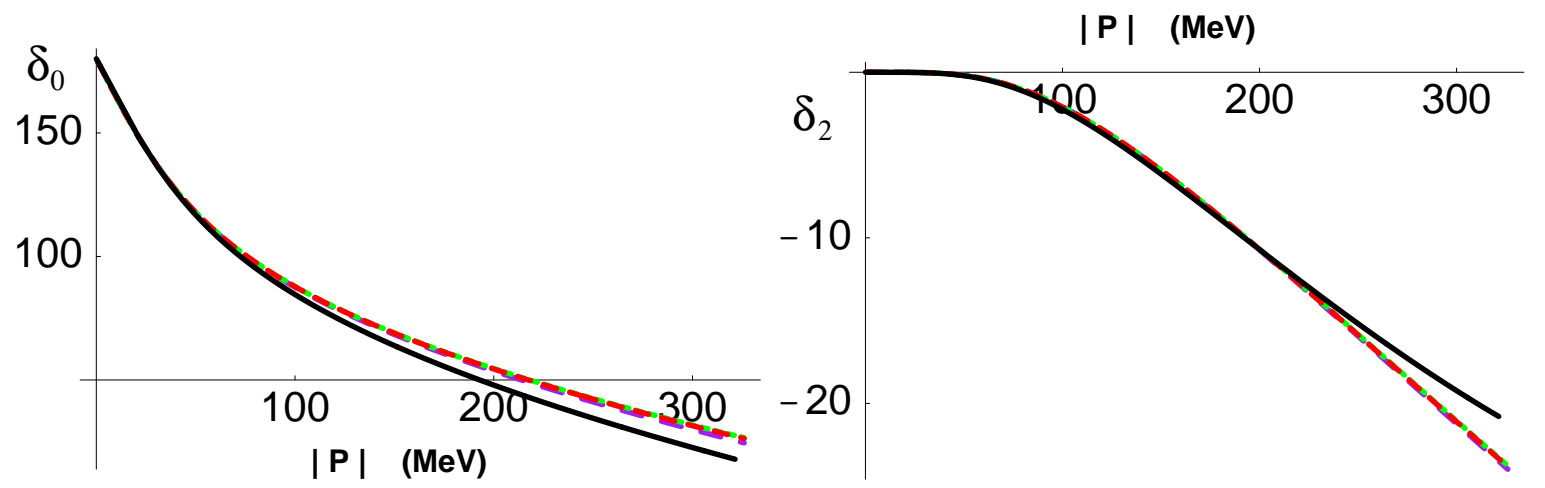

Figure 5: The ${ }^{3} S_{1}-{ }^{3} D_{1}$ phase-shifts as a function of the CoM momentum. The solid line is the Nijmegen phase-shift analysis [17]. The long-dash line corresponds to $R=0.45 \mathrm{fm}$ $(\Lambda=438 \mathrm{MeV})$, the medium-dash line corresponds to $R=0.21 \mathrm{fm}(\Lambda=938 \mathrm{MeV})$, and the dotted line corresponds to $R=0.10 \mathrm{fm}(\Lambda=1970 \mathrm{MeV})$. The phase-shifts $\delta_{0}$ and $\delta_{2}$ are clearly cutoff-independent (see text).

The scaling of the leading explicit chiral symmetry breaking operators in the UV can be found by perturbatively inserting the difference between the OPE potential for $m_{q} \neq 0$

\footnotetext{
${ }^{6}$ For sufficiently small coupling, analytic behavior in $\alpha_{\pi}$ is recovered, as detailed in Ref. [15].
} 


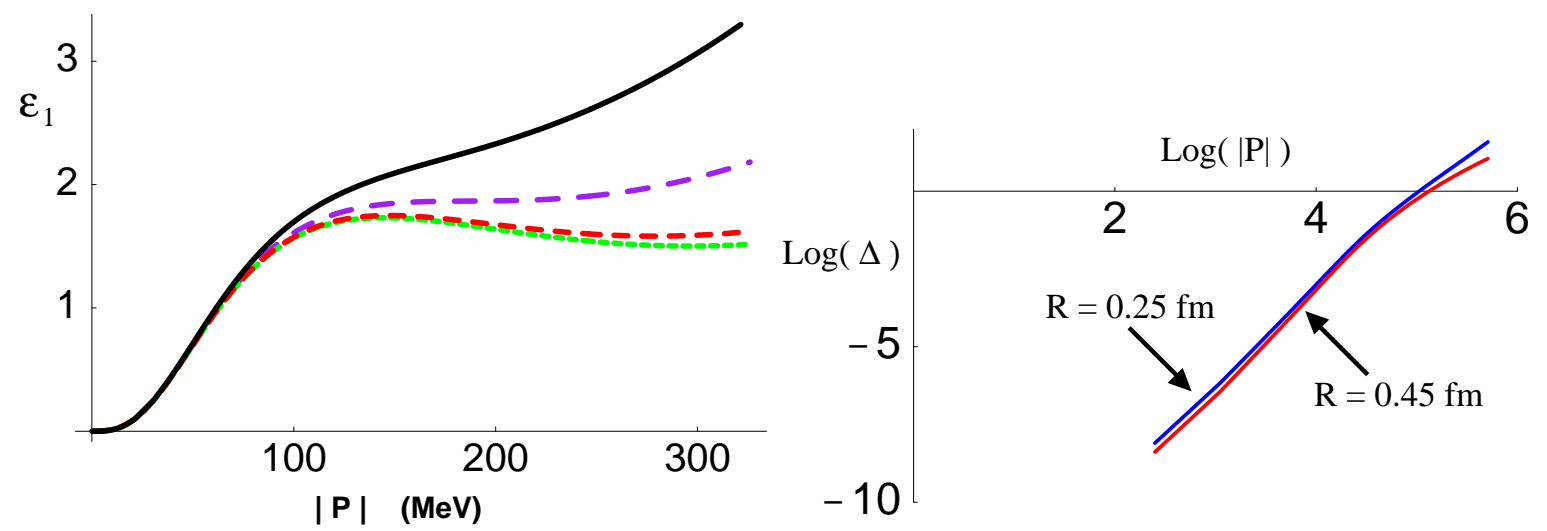

Figure 6: The left panel shows $\varepsilon_{1}$ as a function of the CoM momentum.. The solid line is the Nijmegen phase-shift analysis [17]. The long-dash line corresponds to $R=0.45 \mathrm{fm}$ $(\Lambda=438 \mathrm{MeV})$, the medium-dash line corresponds to $R=0.21 \mathrm{fm}(\Lambda=938 \mathrm{MeV})$, and the dotted line corresponds to $R=0.10 \mathrm{fm}(\Lambda=1970 \mathrm{MeV})$. The right panel shows an error plot for $\varepsilon_{1}$. Momentum $|p|$ is in units of $\mathrm{MeV}$. The quantity $\Delta$ is the difference between the EFT calculation discussed in the text and the Nijmegen phase-shift analysis. The slopes of the curves for $R=0.25 \mathrm{fm}$ and $R=0.45 \mathrm{fm}$ are $\sim 2$.

and its value in the chiral limit,

$$
\left\langle\psi^{\prime}\left|V\left(m_{\pi}\right)-V(0)\right| \psi\right\rangle \sim g_{A}^{2} A^{2} \int_{R}^{\infty} d r r^{\frac{3}{2}} \cos ^{2}\left[2 \sqrt{\frac{6 \alpha_{\pi} M}{r}}\right] \frac{m_{\pi}^{2}}{r} \sim-g_{A}^{2} A^{2} m_{\pi}^{2} R^{\frac{3}{2}}+\ldots
$$

where the ellipses denote terms that are finite as $R \rightarrow 0$. From the momentum-space perspective these leading explicit-breaking effects have a strange $\Lambda_{\chi}^{-3 / 2}$ scaling where $\Lambda_{\chi}$ is the chiral symmetry breaking scale. In practice, $R$ is kept finite and while one might consider putting in a $D_{2}$ operator in order to remove this $R$ dependence, it is formally not necessary. This is in contrast to the ${ }^{1} S_{0}$ channel, where logarithmic dependence is found, eq. (10), which does require the inclusion of a $D_{2}$ operator.

\section{$3.2{ }^{3} S_{1}-{ }^{3} D_{1}$ Phase-Shifts Perturbatively in $V\left(r ; m_{\pi}\right)-V(r ; 0)$}

There is now reason to believe that a perturbative expansion about the chiral limit is formally consistent in both the ${ }^{1} S_{0}$ and ${ }^{3} S_{1}-{ }^{3} D_{1}$ channels. In order for this expansion to be useful it must also converge. At NLO there will be finite $\mathcal{O}\left(m_{\pi}^{2}\right)$ corrections from the perturbative expansion in $V\left(r ; m_{\pi}\right)-V(r ; 0)$. However, since $\alpha_{\pi}$ is itself an expansion about the chiral limit, the $D_{2}$ operator will contribute distinctly from the $C_{0}$ operator. Hence, knowledge of $D_{2}$ requires a distinct measurement involving multi-pion interactions with nucleons. In the absence of knowledge about $D_{2}$, a well-defined, and significant computation that can be performed is the perturbative expansion of the scattering amplitudes 
in $V\left(r ; m_{\pi}\right)-V(r ; 0)$, the difference between the OPE potential evaluated at the physical value of $m_{\pi}$ and the OPE potential for a massless pion. Unfortunately, this does not correspond to the chiral limit, as the couplings such as $g_{A}$ and $F_{\pi}$ are held fixed at their physical values [. The STMoNP suggests that one should expect the perturbative phase-shifts to be most deformed at lower-momenta, and approach the observed value at higher momenta. Fig. 7 shows the phase-shifts and mixing angle of the ${ }^{3} S_{1}-{ }^{3} D_{1}$ coupled channels order-by-order in perturbation theory out to $\mathrm{N}^{6} \mathrm{LO}$. The expansion is convergent $\mathrm{P}$, but only slowly. Despite the relatively poor convergence, this result is important

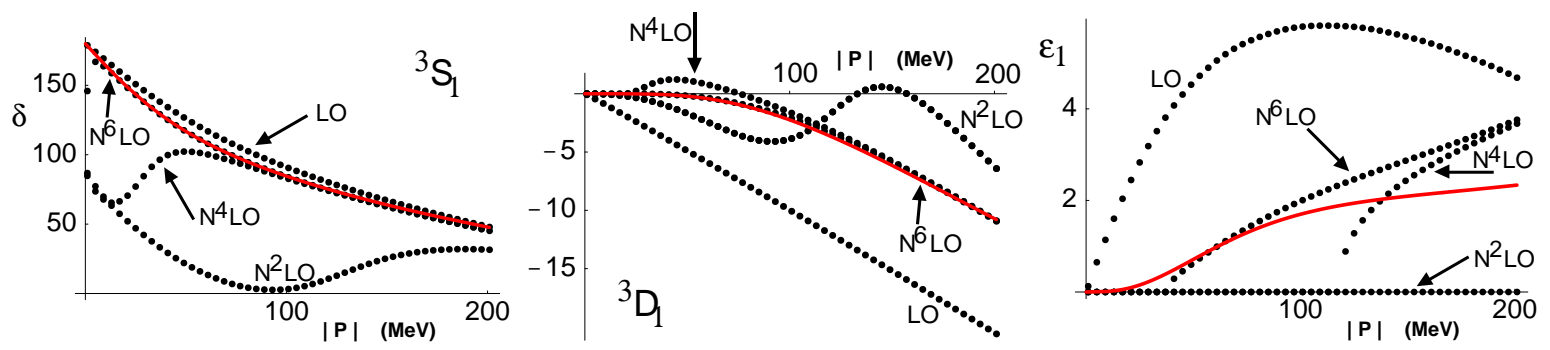

Figure 7: The $\delta_{0}, \delta_{2}$ phase-shifts and $\varepsilon_{1}$ mixing angle in perturbation theory out to $N^{8} L O$ in $V\left(r ; m_{\pi}\right)-V(r ; 0)$. The solid line is the result of the Nijmegen phase-shift analysis [17], while the dotted curves correspond to the $L O, N^{2} L O, N^{4} L O$ and $N^{6} L O$ results, as indicated on each panel. We have not shown odd orders to reduce clutter. These results were obtained for a square well of radius of $R=0.25 \mathrm{fm}$, with an energy-independent squarewell depth of $M V_{0}=1.01 \times 10^{6} \mathrm{MeV}^{2}$ and $M V_{2} k^{2}=23.54 k^{2}$.

as it demonstrates that the expansion of the potential about the chiral limit is convergent. Clearly, one must be concerned about the slow convergence of this expansion. One hopes that this will not seriously impact the construction of a systematic chiral expansion, but unfortunately it may.

\section{How Nuclear Forces Depend on $m_{q}$}

One of the important goals of the EFT investigations is to obtain the dependence of nuclear observables upon the masses of the up and down quarks, and eventually the strange quark. This would allow one to determine the deuteron binding energy in the chiral limit, and other interesting but perhaps somewhat academic features of chiral-limit nuclear physics. From a practical point of view, knowledge of the quark-mass dependence of observables will allow one to isolate terms in the chiral Lagrangian that contribute differently to elastic

\footnotetext{
${ }^{7}$ An expansion in $V\left(r ; m_{\pi}\right)$ (not subtracting the $V(r ; 0)$ ) should recover the FMS analysis of KSW power counting at $\mathrm{N}^{2} \mathrm{LO}[10]$ minus the contributions from radiation pions.

${ }^{8}$ By convergent, we mean that the perturbative expansion converges to the solution to the Schrödinger equation for the full potential $V\left(r ; m_{\pi}\right)$.
} 
and inelastic processes involving pions. This is an important step toward developing a unified theory of elastic and inelastic processes. Further, knowledge of the quark-mass dependence of these observables will provide the bridge between proposed lattice-gauge theory calculations, performed at quark masses much larger than the physical values, and nature $\mathrm{f}$.

In multi-nucleon sector calculations, quark-mass dependences are both explicit and implicit. For instance, in the NN-sector there is explicit $m_{q}$-dependence in the mass of the pion in the nuclear potential and also in four-nucleon operators. However, there is implicit $m_{q}$-dependence in the couplings in the meson sector (e.g. $F_{\pi}$ ) and single-nucleon sector (e.g. $\left.g_{A}\right)$. Without measuring processes involving either initial or final-state pions, the coefficient of each four-nucleon operator that has explicit $m_{q}$ dependence cannot be determined. Therefore, what follows is for illustration only, and we do not claim to presently know the deuteron binding energy, or any other finely-tuned observable in the chiral limit.

\subsection{Explicit $m_{q}$-Dependence only}

It is interesting to know the behavior of observables in nuclear systems as a function of the explicit factors of $m_{q}$ that appear in the nuclear potential. That is to say, the value of the pion mass in the OPE potential scales as $\sim m_{q}^{1 / 2}$ but other quantities, such as $F_{\pi}, M$ and $g_{A}$ are fixed to their measured values. It is this scenario that can be directly compared with all potential-model calculations, where the implicit $m_{q}$-dependence of the short-distance part of the nuclear interaction is unknown.

As we demonstrated earlier, with square-well regularization of the ${ }^{3} S_{1}-{ }^{3} D_{1}$ coupled channels, the $m_{q}$-dependent four-nucleon counterterm required to yield a renormalizationscale $(R)$ independent result scales like $D_{2} \sim R^{3 / 2}$ as $R \rightarrow 0$, and therefore is naively higher order in the power counting. Hence, we may vary the value of the explicit factors of $m_{\pi}$ in the nuclear potential without an accompanying modification to the depth of the regulating square well. A plot of the deuteron binding energy as a function of $m_{\pi}$ resulting from numerically solving the Schrödinger equation is shown in fig. 8. For the physical value of the pion mass we recover the deuteron binding energy to reasonable accuracy, $B_{d}=2.211 \mathrm{MeV}$ (essentially independent of $R$ ). In the chiral limit one finds that the deuteron is bound by $B_{d}^{0} \sim 4.2 \mathrm{MeV}$. This value is still somewhat small compared to $F_{\pi}^{2} /(2 M) \sim 10 \mathrm{MeV}$, that one might expect to arise in QCD, and therefore one would conclude that the deuteron is still weakly bound in the chiral limit! Our calculation of the deuteron binding energy in the chiral limit agrees with that obtained with the AV18 potential [31] of $B_{d}^{0,(A V 18)} \sim 4.1 \mathrm{MeV}$. These very-similar results are in qualitative agreement (but of opposite sign) with a previous estimate [32] based on a first-order

\footnotetext{
${ }^{9}$ Recently, remarkable progress has been made in understanding how to compute higher-order coefficients of the chiral Lagrangian describing the pseudo-Goldstone bosons [29]. Partially-quenched lattice calculations have been matched onto a partially-quenched chiral Lagrangian and combinations of the Gasser-Leutwyler coefficients [30], the $L_{i}$ 's, have been determined. At this point in time, a partiallyquenched EFT describing the single and multi-nucleon sectors does not exist.
} 


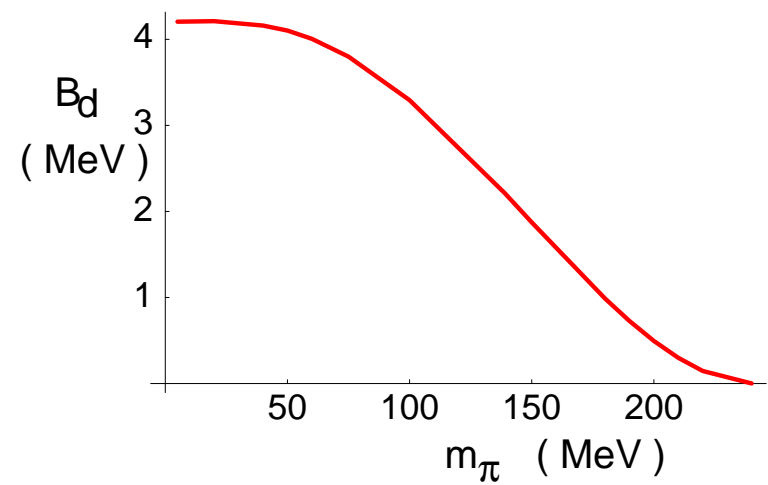

Figure 8: The deuteron binding energy as a function of the $m_{\pi}$ that explicitly appears in the OPE potential. For $R=0.25 \mathrm{fm}$, an energy-independent square well depth of $M V_{0}=$ $1.01 \times 10^{6} \mathrm{MeV}^{2}$ and a square well that depends linearly on energy of $M V_{2} k^{2}=23.54 k^{2}$ reproduce the scattering length and effective range in the ${ }^{3} S_{1}$ channel for $m_{\pi}=139 \mathrm{MeV}$.

perturbative calculation with wavefunctions from the Paris potential and Bonn potential.

In addition to the deuteron binding energy, one gains useful insight into the chiral limit of the NN-system by examining the phase-shifts and mixing parameters of the ${ }^{3} S_{1}-{ }^{3} D_{1}$ coupled channels. We have considered the perturbative expansion of the phase-shifts and mixing angle previously, but it is worth restating in this discussion. By numerically solving

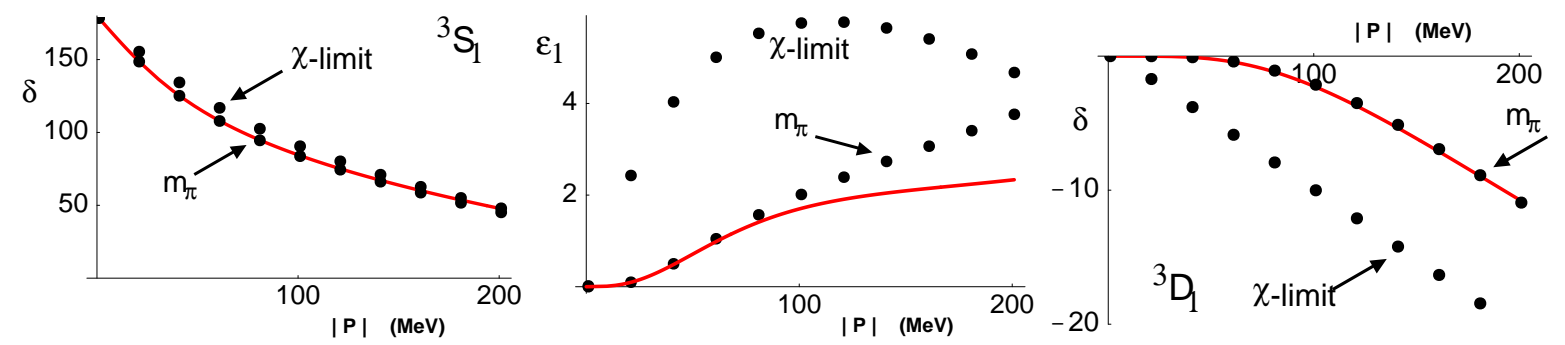

Figure 9: The phase-shifts $\delta_{0}, \delta_{2}$ and the mixing parameter $\varepsilon_{1}$ of the ${ }^{3} S_{1}-{ }^{3} D_{1}$ coupled channels, generated by the parameter set as fig. 8. The solid curves correspond to the Nijmegen phase-shift analysis [17], while the two sets of dotted curves correspond to our calculated result for the physical value of the pion mass, denoted by $m_{\pi}$ in the plots, and the chiral limit, as denoted in the plots.

the Schrödinger equation we find the phase-shifts and mixing parameter shown in fig. 9, see also fig. 7. It is clear that the observed ${ }^{3} S_{1}$ phase-shift does not differ significantly from that resulting from taking $m_{\pi}$ to zero in the potential. However, as expected, the ${ }^{3} D_{1}$ phase-shift and mixing parameter $\varepsilon_{1}$ differ significantly from this "chiral limit". As 
the deuteron is bound largely by the strong attractive tensor force, the observed increase in $\varepsilon_{1}$ explains the deuteron binding energy computed above.

\subsection{Implicit and Explicit $m_{q}$-Dependence}

In addition to the explicit $m_{q}$ dependence of the OPE, there is implicit $m_{q}$-dependence, as the couplings in the meson and single-nucleon sector also depend upon $m_{q}$ at subleading orders. Such dependences have been computed previously [33 for QCD with $S U(2)_{L} \otimes$ $S U(2)_{R}$ chiral symmetry. The physical values of the pion decay constant $F_{\pi}$, the nucleon mass $M$ and the axial coupling constant $g_{A}$ are given in terms of the pion mass at lowest order in the chiral expansion $m$, and $F, M_{0}$ and $g$, their respective values in the chiral limit

$$
\begin{aligned}
F_{\pi} & =F\left[1+\frac{m^{2}}{16 \pi^{2} F^{2}} \bar{l}_{4}+\mathcal{O}\left(m^{4}\right)\right] \\
M & =M_{0}-4 m^{2} c_{1}+\mathcal{O}\left(m^{3}\right) \\
g_{A} & =g\left[1-\frac{2 g^{2}+1}{16 \pi^{2} F^{2}} m^{2} \log \left(\frac{m^{2}}{\lambda^{2}}\right)+\mathcal{O}\left(m^{2}\right)\right] .
\end{aligned}
$$

where $\bar{l}_{4}=4.4 \pm 0.2$ [33, 34], $c_{1} \sim-1 \mathrm{GeV}^{-1}$ [35], and we have use $m=140 \mathrm{MeV}$. We have retained only the leading chiral-logarithmic contribution to $g_{A}$, and have chosen a renormalization scale of $\lambda=500 \mathrm{MeV}$. In addition to these relations, there is also $m_{q^{-}}$ dependence in the relation between the $\pi N N$ coupling and the axial matrix element, as computed at LO in Ref. [36],

$$
g_{\pi N N}=\frac{g_{A} M}{F_{\pi}}\left(1-2 \frac{m_{\pi}^{2}}{g_{A}} \bar{d}_{18}\right),
$$

where we will use $\bar{d}_{18} \sim-1.0 \mathrm{GeV}^{-2}$ 36]. Even with this set of $m_{q}$-dependences included into the calculation, there very well could remain uncalculated $m_{q}$-dependences. In particular, the implicit $m_{q}$-dependences modify the strength of the chiral-limit OPE potential. This will give rise to a stronger $\mathrm{R}$-dependence in the local four-nucleon $m_{q}$-dependent operator with coefficient $D_{2}$. Until processes involving external pions are computed, the contribution of this operator cannot be isolated. Therefore, for demonstrative purposes only, we do not modify the depth of the square wells evaluated for physical values of $m_{\pi}$ in our chiral extrapolation.

The behavior of the deuteron binding energy as a function of $m_{\pi}$ when both the implicit and explicit dependences are included is shown in fig. 10. In this scenario the deuteron binding energy decreases as the chiral limit is approached, and the deuteron is unbound for $m_{\pi} \lesssim 100 \mathrm{MeV}$. This is precisely the opposite $m_{\pi}$-dependence to that found when only the explicit dependence is retained. Therefore, at this point in time a reliable calculation of the $m_{\pi}$-dependence of the deuteron binding energy cannot be performed. It should 


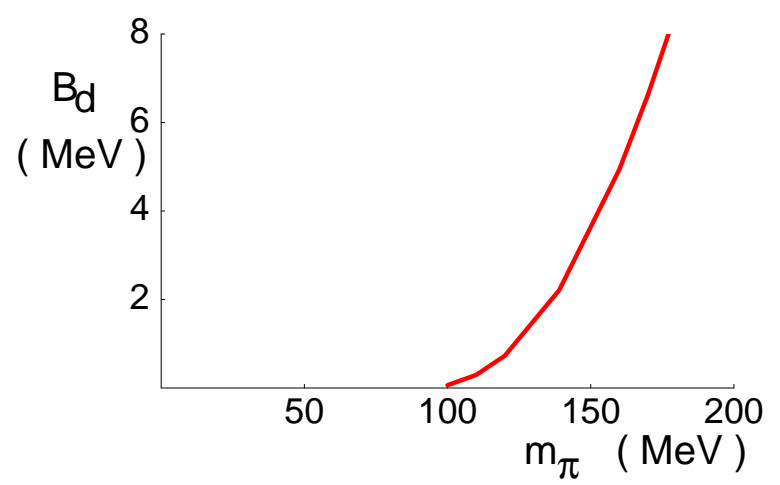

Figure 10: The deuteron binding energy as a function of the pion mass that enters both explicitly and implicitly in the nuclear potential. For a radius of $R=0.25 \mathrm{fm}$, an energyindependent square-well depth of $M V_{0}=1.03 \times 10^{7} \mathrm{MeV}^{2}$ and a square well that depends linearly on energy of $M V_{2} k^{2}=15.25 k^{2}$ reproduce the scattering length and effective range in the ${ }^{3} S_{1}$ channel for the physical value of $m_{\pi}=139 \mathrm{MeV}$.

come as no surprise that the fine-tuning in this channel is significantly disturbed by what would appear to be higher-order contributions. Their impact is strongly enhanced by the large cancellation between the kinetic and potential energy of the near-threshold bound state.

The ${ }^{3} S_{1}-{ }^{3} D_{1}$ phase-shifts and mixing parameter are shown in fig. 11 for this scenario. As required, the ${ }^{3} S_{1}$ phase-shift is very different in this chiral limit, due to the fact that
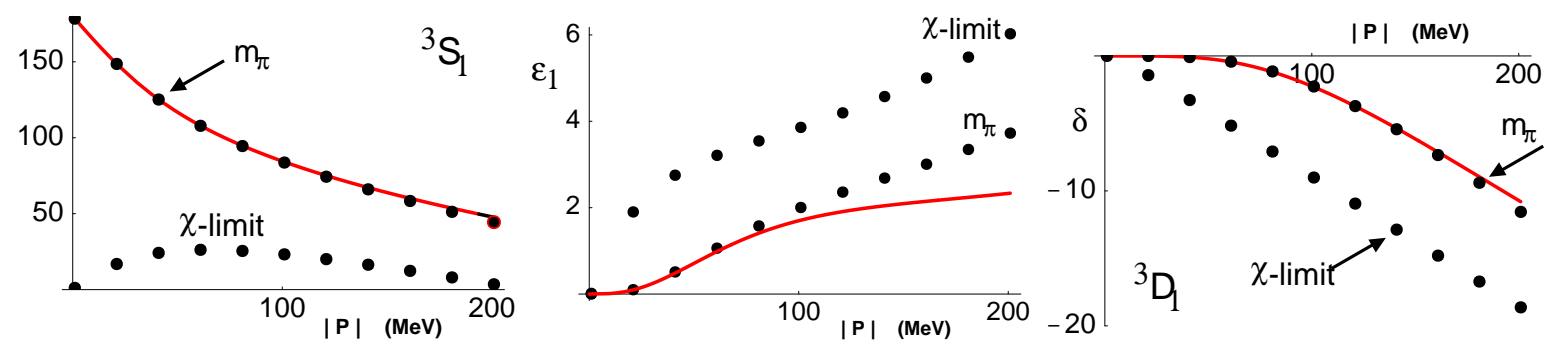

Figure 11: The phase-shifts $\delta_{0}, \delta_{2}$ and the mixing parameter $\varepsilon_{1}$ of the ${ }^{3} S_{1}-{ }^{3} D_{1}$ coupled channels, generated by the parameter set as fig. 10. The solid curves correspond to the Nijmegen phase-shift analysis [17], while the two sets of dotted curves correspond to our calculated result for the physical value of the pion mass, denoted by $m_{\pi}$ in the plots, and the chiral limit, as denoted in the plots.

there is no bound state. On the other hand, the ${ }^{3} D_{1}$ phase-shift is very similar to that computed with only explicit $m_{\pi}$-dependence retained. Clearly, the $m_{q}$-dependence of the 
OPE couplings makes a perturbatively small contribution to this scattering amplitude, as one might expect (there is no pole in this channel).

\subsection{Is an Expansion about the Chiral Limit Sensible?}

We have made the case that an EFT can be developed by performing an expansion about the chiral limit. Such an expansion overcomes the formal problem with W power counting, that KSW power counting successfully resolved in the ${ }^{1} S_{0}$ channel. However, given that the deuteron may be unbound in the chiral limit, it is natural to ask whether such an expansion makes any sense. The existence or non-existence of the bound state depends upon subleading contributions to the scattering amplitude. That is to say, small changes in couplings have large effects due to the inherent fine-tuning in the s-wave channels.

Therefore, in the ${ }^{3} S_{1}-{ }^{3} D_{1}$ coupled channels it may be consistent to perform a chiral expansion of observables about a deuteron wavefunction defined at high orders in the expansion, as has long been advocated by several groups (see Ref. [6, 37]) who have shown that many observables are relatively insensitive to the choice of regularization. For deuteron observables, the EFT would then become an expansion of the interaction kernel, up to the order at which the wavefunction is consistently defined. Order by order, observables would be independent of $R$ as $R \rightarrow 0$ if the calculation is performed correctly.

\subsection{Matching to Numerical Results from Lattice-QCD}

In order to match to any foreseeable lattice-QCD results, an extrapolation in $m_{q}$ will be required. What we have presented in this work is the framework required to match to a fully unquenched lattice computation, and we have shown that there is currently no control of the extrapolation away from the physical value of $m_{\pi}$, due to the fine-tunings that exist in nature. However, future lattice computations are likely to be partially-quenched [29], as opposed to unquenched. Therefore, a partially-quenched EFT will need to be constructed in order to correctly perform the $m_{q}$-extrapolation from the lattice masses, to the physical masses. Given our relatively primitive understanding of the EFT for QCD, and also considering that the partially-quenched EFT required to perform extrapolations in the single-nucleon sector does not exist at this point in time, a significant theoretical effort is required to put in place a framework that will enable future lattice-QCD computations to make rigorous statements about multi-nucleon systems.

It is exciting to note that attempts have been made to compute NN scattering parameters with quenched lattice-QCD. Ref. [38] computes the ${ }^{1} S_{0}$ and ${ }^{3} S_{1}$ scattering lengths in quenched QCD at $\beta=5.7$ with Wilson quarks using Lüscher's finite-volume algorithm, which expresses the energy of a two-particle state as a perturbative expansion in the scattering length divided by the size of the box [39]. Practical computations require a sufficiently small box which in turn should be larger than the scattering length. Hence Lüscher's method is ideal for systems with "natural" scattering lengths, that is with size of order a characteristic physical length scale. Of course in the NN system, the scattering lengths are much larger than the characteristic physical length scale given by 
$\Lambda_{Q C D}^{-1}$. Nevertheless one might suppose that away from the physical value of the pion mass, the scattering lengths relax to natural values, thus allowing their determination from the lattice. In fig. 12 we exhibit the ${ }^{3} S_{1}$ scattering length as a function of the pion mass as compared to the lattice data from Ref. [38]. The left panel has only negligible cutoff dependence, by construction. The cutoff dependence exhibited in the right panel demonstrates the incomplete nature of the calculation with implicit pion mass dependence (the absence of $D_{2}$ ). We truncate our curves at $\sim 400 \mathrm{MeV}$ as higher-order effects are increasingly important in this region.
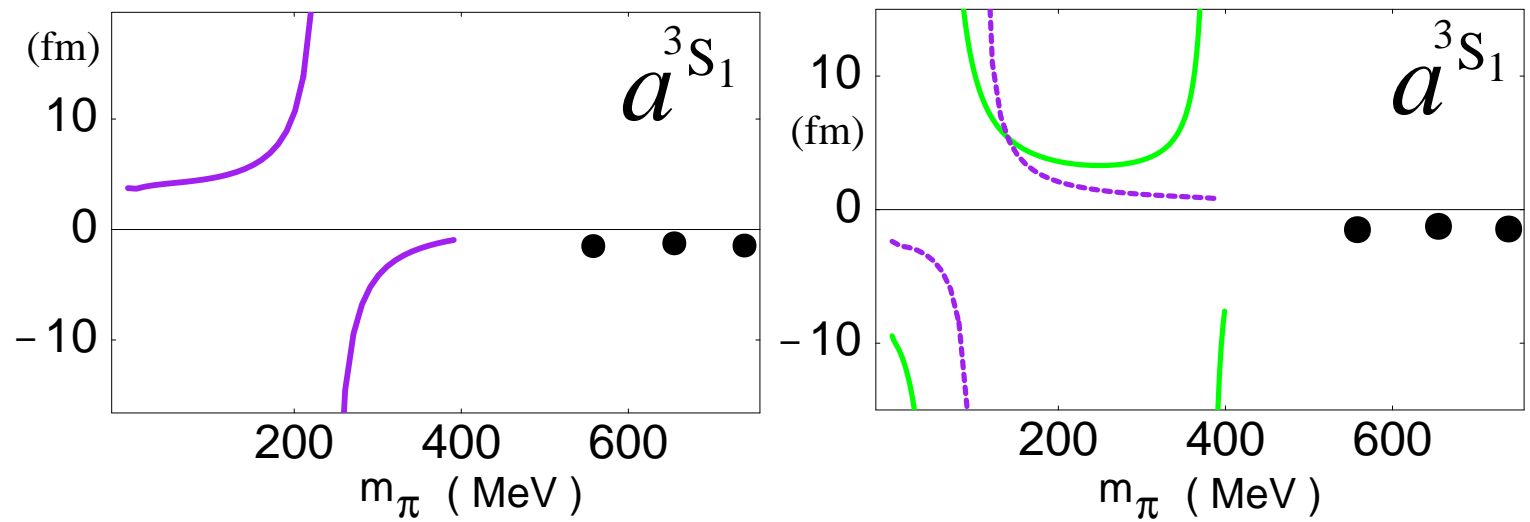

Figure 12: The ${ }^{3} S_{1}$ scattering length as a function of $m_{\pi}$. In the left panel, the quantities $g_{A}, M$ and $F_{\pi}$ are set equal to their physical values for all $m_{\pi}$. In the right panel, the $L O m_{\pi}$ dependence of $g_{A}, M$ and $F_{\pi}$ is included. The dashed (solid) line corresponds to a radius of $R=0.25 \mathrm{fm},(0.45 \mathrm{fm})$ and an energy-independent square-well depth of $M V_{0}=-1.04 \times 10^{4} \mathrm{MeV}^{2}\left(-7.82 \times 10^{6} \mathrm{MeV}^{2}\right)$ which reproduce the scattering length for the physical value of $m_{\pi}=139 \mathrm{MeV}$. The dots are lattice QCD data, as explained in the text.

\section{Conclusions and Thoughts}

We have addressed several important issues concerning the development of a consistent and converging EFT to describe multi-nucleon systems. Chiral perturbation theory in the single-nucleon sector is a special case of a more general ordering of operators. In the presence of more than one nucleon, the momentum expansion is nonperturbative at some level to accommodate the fine-tuned scales, while the $m_{q}$-expansion remains perturbative. In other words, the perturbative expansion of nuclear forces is an expansion about the chiral limit. In the ${ }^{1} S_{0}$ channel only local operators are treated nonperturbatively, whereas in the ${ }^{3} S_{1}-{ }^{3} D_{1}$ coupled channels it is necessary to resum the non-local, singular part of OPE which survives in the chiral limit. 
The amount of chiral-limit physics which must be resummed is determined phenomenologically channel-by-channel. One can always choose to resum the entire momentum expansion since perturbative physics remains so even when treated nonperturbatively. KSW power counting is the minimal power-counting scheme to describe systems with unnaturally-large scattering lengths; i.e. one operator in the momentum expansion is summed to all orders. If the effective range is also unnaturally large, it too should be resummed and this in turn constitutes a legitimate ordering of operators. W power counting is a prescription which sums a kernel computed to a given order in chiral perturbation theory to all orders. Here there is a subtlety; one must ensure that at a given order in the expansion there is a correspondence between divergences and the local operators which absorb them. This is obeyed by $\mathrm{W}$ power counting in the ${ }^{3} S_{1}-{ }^{3} D_{1}$ coupled channels but not in the ${ }^{1} S_{0}$ channel. A bizarre feature that emerges from the nonperturbative nature of the ${ }^{3} S_{1}-{ }^{3} D_{1}$ coupled channels is the scaling of operators with fractional powers of the chiral symmetry-breaking scale, e.g. $D_{2} \sim \Lambda_{\chi}^{-3 / 2}$

Our partial higher-order calculations in the ${ }^{3} S_{1}-{ }^{3} D_{1}$ coupled channels suggest that an expansion about the chiral limit will converge. However, a full NLO calculation is required in order to make a more definite conclusion and to give meaningful predictions for the deuteron binding energy in the chiral limit. The use of the square well as a short-distance regulator has proved valuable in giving analytic formulas for the RG running of the counterterms. However, the necessity of computing processes with external gauge fields suggests use of a regulator that manifestly respects gauge invariance, like dimensional regularization or Pauli-Villars. An intriguing puzzle remains concerning the relationship between square-well regularization and the matching of delta-function interactions to singular potentials. In taking the limit $R \rightarrow 0$ one finds that the singular potential wavefunctions vanish. This makes it difficult to understand how a delta-function interaction can modify the physical asymptotic phase. This puzzle is compounded in the coupled-channel system.

An EFT with dynamical pions is required to describe both elastic and inelastic processes involving momenta greater than $\sim m_{\pi}$. For very-low momentum processes $\ll m_{\pi}$ it is appropriate and consistent to work with $\operatorname{EFT}(\not)$. All quark-mass dependence is implicit in the coefficients of local operators as chiral symmetry is not a symmetry of this EFT.

One may worry that observables traditionally used to probe nuclear forces, like the electric-quadrupole moment, will not be well reproduced by our proposed EFT and will be cutoff dependent. However contributions from gauge-invariant, four-nucleon, onephoton operators that arise at higher order in the EFT will precisely compensate the cutoff dependence and when combined with the one-body contributions from the nuclear wave function will reproduce these observables [40].

Finally, there is a significant overlap between the approach advocated in this paper, and a more mechanical approach to the problem. Haxton and collaborators 41 have shown that the nuclear Shell Model can be formulated as an effective theory, and its convergence can be dramatically improved by resumming the "short-distance" part of the NN interaction and then performing perturbation theory with the remaining "longdistance" part. The separation into long- and short-distance is defined numerically by 
the shape of the NN potential channel-by-channel, rather than by underlying hadronic structure. Nonetheless this approach recovers the essential parts of the expansion we are advocating in this work.

\section{Acknowledgements}

We thank W. Haxton, U. Meißner, D. Phillips, G. Rupak, S. Sharpe, D. Sprung, R. Wiringa and M. Wise for useful discussions. This research was supported in part by the DOE grant DE-FG03-97ER41014 (SRB,MJS), and by the Director, Office of Energy Research, Office of High Energy and Nuclear Physics, Division of Nuclear Physics, and by the Office of Basic Energy Science, Division of Nuclear Science, of the U.S. Department of Energy under Contract No. DE-AC03-76SF00098 (PFB), and by RIKEN, Brookhaven National Laboratory and the U.S. Department of Energy DE-AC02-98CH10886 (UvK).

\section{A The Standard Toy Model of Nuclear Physics: Three Yukawas}

One can gain significant insight into the perturbative structure of the ${ }^{1} S_{0}$ channel and ${ }^{3} S_{1}-{ }^{3} D_{1}$ coupled channels by studying the behavior of a toy theory of nucleons interacting in a potential formed from three Yukawa exchanges [16]. The potential has the form

$$
\begin{aligned}
V(r) & =V_{s}(r)+V_{\pi}(r) \\
V_{s}(r) & =\alpha_{\rho} \frac{e^{-m_{\rho} r}}{r}-\alpha_{\sigma} \frac{e^{-m_{\sigma} r}}{r}, V_{\pi}(r)=-\bar{\alpha}_{\pi} \frac{e^{-m_{\pi} r}}{r},
\end{aligned}
$$

where the masses

$$
m_{\rho}=770 \mathrm{MeV}, m_{\sigma}=500 \mathrm{MeV}, m_{\pi}=140 \mathrm{MeV} \text {, }
$$

and couplings

$$
\alpha_{\rho}=14.65, \alpha_{\sigma}=7.00, \bar{\alpha}_{\pi}=0.075
$$

are chosen, along with a nucleon mass of $M=940 \mathrm{MeV}$ to approximately reproduce both the scattering length, and effective range in the ${ }^{1} S_{0}$ channel, $a^{3 Y}=-23.7 \mathrm{fm}$ and $r^{3 Y}=2.57 \mathrm{fm}$. In making contact with OPE in the real world, the coupling $\bar{\alpha}_{\pi}$ depends upon both the pion mass, $m_{\pi}$, and the axial coupling constant, $g_{A}, \bar{\alpha}_{\pi} \propto g_{A}^{2} m_{\pi}^{2}$. Therefore, perturbing in the pion potential is equivalent to perturbing in either $g_{A}, m_{\pi}$ or both. However, it is convenient to think about expanding about the chiral limit, i.e. expanding in $\delta V=V\left(r ; m_{\pi}\right)-V(r ; 0)=V_{\pi}$. One significant difference between this toy theory and the real world is that $M$ does not receive contributions from "potential pion" loops, and 
so $M=940 \mathrm{MeV}$ at each order in perturbation theory. This will not be the case in the real world, where $M$ depends analytically and non-analytically on the quark masses.

Solving the Schrödinger equation in perturbation theory is straightforward. The s-wave radial wavefunction, $U_{3 Y}^{\text {full }}(r)$, satisfies

$$
\frac{1}{M} \frac{d^{2}}{d r^{2}} U_{3 Y}^{\text {full }}-(V-E) U_{3 Y}^{\text {full }}=0 .
$$

Writing $U_{3 Y}^{\text {full }}=U_{3 Y}^{0}+U_{3 Y}^{1}+\ldots$, where the superscript denotes the number of insertions of $V_{\pi}$, it follows that

$$
\begin{aligned}
& \frac{1}{M} \frac{d^{2}}{d r^{2}} U_{3 Y}^{0}-\left(V_{s}-E\right) U_{3 Y}^{0}=0 \\
& \frac{1}{M} \frac{d^{2}}{d r^{2}} U_{3 Y}^{j}-\left(V_{s}-E\right) U_{3 Y}^{j}=V_{\pi} U_{3 Y}^{j-1}, \quad j \geq 1
\end{aligned}
$$

with boundary conditions at the origin chosen appropriately for each of the $U_{3 Y}^{j}$. The s-wave phase-shifts $\delta_{3 Y}$ can be found from the wavefunction at any given order in perturbation theory by simply projecting out the incoming and outgoing asymptotic plane waves, e.g. see Ref. [8]. This method automatically provides a unitary phase-shift as one is expanding $\exp (i 2 \delta)$ which necessarily involves $V_{\pi}$ to all orders. We denote this expansion as $E 1$. One may perform a further expansion of $\delta_{3 Y}$ to any given order in $V_{\pi}$ that is unitary to a given order in $g_{A}^{2}$, and we denote this expansion as $E 2$.

At LO, corresponding to no insertions of $V_{\pi}$, the "short-distance" phase-shifts resulting from $E 1$ and $E 2$ are identical, as shown in fig. 13. One sees sizable deviations between

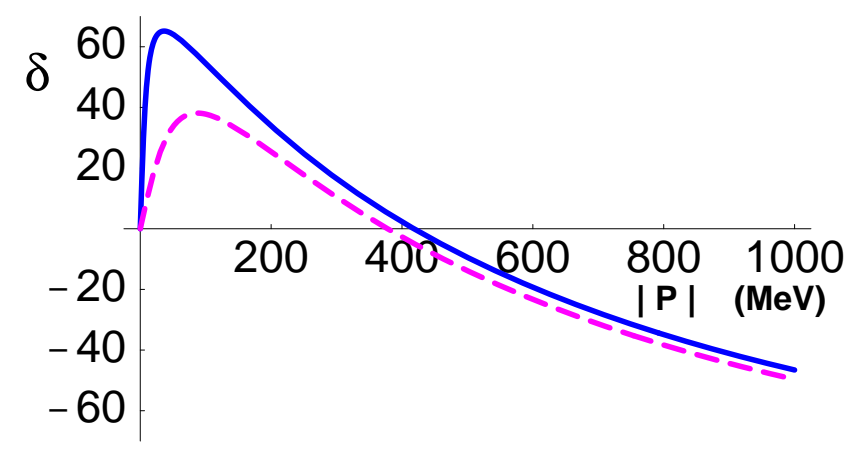

Figure 13: The full phase-shift (solid) and the phase-shift resulting from zero-insertions of $V_{\pi}$ (dashed) in the three-Yukawa toy model.

the two phase-shifts below $|\mathbf{p}| \lesssim 200 \mathrm{MeV}$. The phase-shifts at higher orders in perturbation theory are shown in fig. 14. It is clear that both expansions converge rapidly from $|\mathbf{p}| \gtrsim 100 \mathrm{MeV}$. Further, expansion $E 1$ converges rapidly for all $|\mathbf{p}|$, while E2 converges somewhat more slowly in the very low $|\mathbf{p}|$ region. 


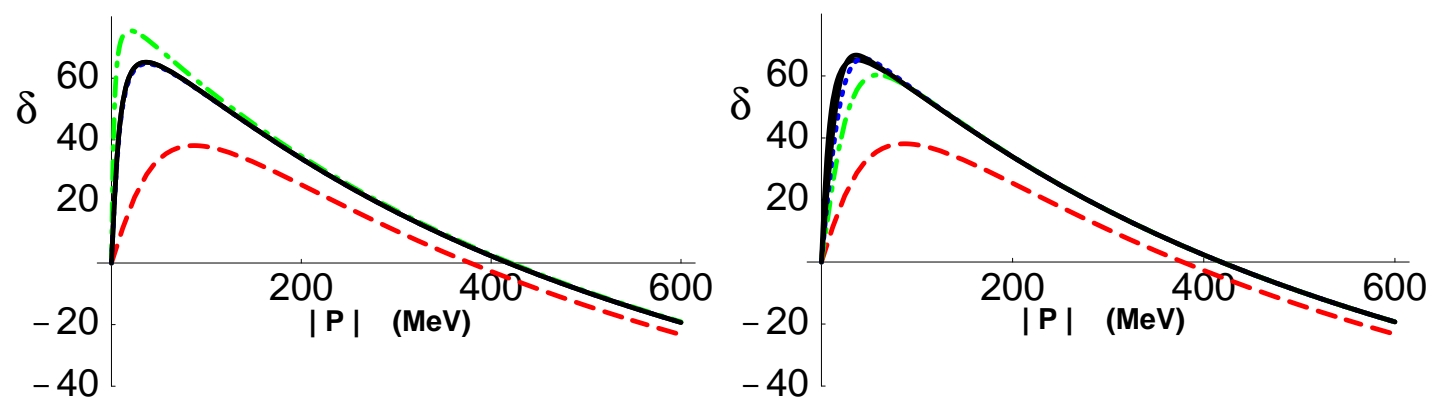

Figure 14: The left panel shows the perturbative phase-shifts in the three-Yukawa toy model obtained by solving the Schrödinger equation, and using expansion E1, while the right panel results from expansion E2. The solid curve is the full phase-shift, while the dashed, dot-dashed and dotted curves correspond to $L O, N L O$, and $N^{2} L O$, respectively. In both cases the $N^{2} L O$ result essentially lies on top of the full result.

While we take some joy in demonstrating the perturbative nature of this theory, it is still the case that we do not know the underlying short-distance physics responsible for the real-world NN interaction and somehow it must be extracted from data. Given that we wish to keep the perturbative aspect of the problem, we need to most efficiently describe the short-distance part of the interaction, whose exact nature we care little about in the low-momentum region. The form of this parameterization has been the source of much debate, i.e. W, KSW or some hybrid. However, for this particular problem we know exactly what we can do to parameterize the short-distance part only, and that is to use effective range theory (ER), which should be valid up to momenta of order $|\mathbf{p}| \sim m_{\sigma} / 2 \sim 250 \mathrm{MeV}$. Therefore, we fit a polynomial of the form

$$
|\mathbf{p}| \cot \delta_{s}=-\frac{1}{a^{s}}+\frac{1}{2} r_{0}^{s}|\mathbf{p}|^{2}+r_{1}^{s}|\mathbf{p}|^{4}+\ldots,
$$

to the "short-distance" phase-shift shown in fig. 13, to find

$$
a^{s}=-3.38 \mathrm{fm}, r_{0}^{s}=2.60 \mathrm{fm}, r_{1}^{s}=0.313 \mathrm{fm}, r_{2}^{s}=0.156 \mathrm{fm} \text {. }
$$

A comparison between the "short-distance" phase-shift, and successively better ER approximations are shown in fig. 15. Retaining only the leading term, i.e. $|\mathbf{p}| \cot \delta_{s}=-\frac{1}{a^{s}}$ is clearly not such a good approximation for momenta $|\mathbf{p}| \gtrsim 40 \mathrm{MeV}$, and corresponds to the underlying perturbative scheme of KSW. However, retaining the short-distance effective range contribution is a significant improvement, but still appears to begin deviating from the true phase-shift for $|\mathbf{p}| \gtrsim 150 \mathrm{MeV}$. However, this is in no way related to $m_{\pi}$, and is a numerical accident. It is this approximation that corresponds to the counting suggested in [16], and fleshed-out in Ref. [19]. As one proceeds to higher orders in the ER expansion, the phase shift is well recovered up to $|\mathbf{p}| \gtrsim 250 \mathrm{MeV}$, as expected. 


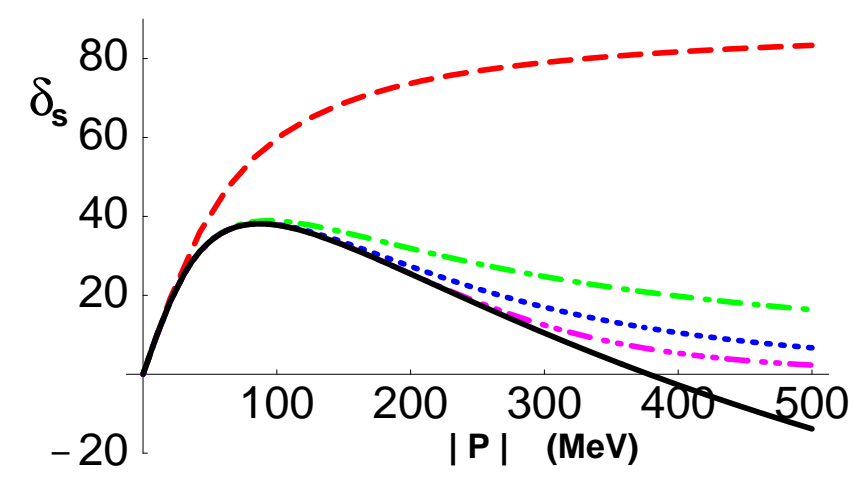

Figure 15: Successive effective-range approximations to the short-distance phase-shift. The solid curve is the exact short-distance phase-shift while the dashed, dot-dashed, dotted and dot-dot-dashed curves correspond to the effective-range expansion including terms up to $1 / a_{s}, r_{0}^{s}, r_{1}^{s}$ and $r_{2}^{s}$, respectively.
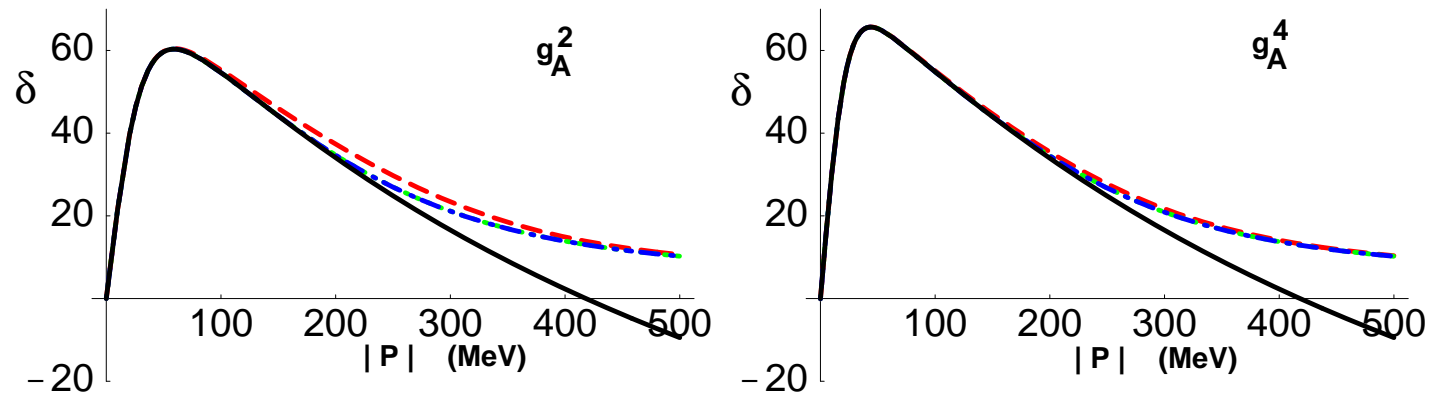

Figure 16: Phase-shifts in the EFT of the three-Yukawa toy model. The dibaryon field describing the short-distance physics is expanded out to order $|\mathbf{p}|^{6}$. The left panel shows the phase-shift up to one insertion of $V_{\pi}$. The solid curve is the full phase-shift at this order obtained from the Schrödinger equation, while the dashed, dot-dashed and dotted curves correspond to one perturbative insertion of $V_{\pi}$ in the EFT expansion including the order $g_{A}^{2}$ counterterms up to $|\mathbf{p}|^{2},|\mathbf{p}|^{4}$ and $|\mathbf{p}|^{6}$, respectively. The right panel shows the phase-shift up to two insertions of $V_{\pi}$. The solid curve is the full phase-shift at this order obtained from the Schrödinger equation, while the dashed, dot-dashed and dotted curves correspond to two perturbative insertions of $V_{\pi}$ in the EFT expansion including the order $g_{A}^{4}$ counterterms up to $|\mathbf{p}|^{2},|\mathbf{p}|^{4}$ and $|\mathbf{p}|^{6}$, respectively. The $g_{A}^{2}$ counterterms determined in the left panel are used in the $\mathcal{O}\left(g_{A}^{4}\right)$ calculation shown in the right panel.

From the EFT point of view, we can use a generalized dibaryon field to include an arbitrary number of terms in the short-distance physics via $|\mathbf{p}| \cot \delta_{s}$. Further, the longdistance amplitudes have been computed out to $\mathrm{N}^{2} \mathrm{LO}$ by Rupak and Shoresh [42]. How- 
ever, there are additional parameters that need to be determined by numerical fitting. They occur because the EFT must be matched to the full theory. The short-distance behavior of graphs involving all three particles, the $\pi, \sigma$ and $\rho$, does not allow for a clean separation into a dibaryon field defined by the short-distance parameters of eq. (29) and pion exchange. However, one can perform the separation into a dibaryon field defined by the short-distance parameters of eq. (29), pion exchange and local finite counterterms of the form $g_{A}^{2 k}\left(m_{\pi} / m_{\sigma}\right)^{n}, g_{A}^{2 k}\left(|\mathbf{p}| / m_{\sigma}\right)^{m}, \ldots$. The LO amplitude in the EFT consists solely of a dibaryon field with the parameters of eq. (29). The NLO amplitude in the EFT consists of the dibaryon field, a single insertion of $V_{\pi}$ and both momentum-independent and momentum-dependent counterterms. As expected, the fit improves as the number of counterterms is increased. It is important to note that the size of the counterterms is determined by $m_{\sigma}$. However, if the $V_{\pi}$ insertion is not included and the counterterms are refit, one finds that their size is now set by $m_{\pi}$, and the overall quality of the fit is noticeably degraded. At $\mathrm{N}^{2} \mathrm{LO}$ the pattern continues with the same dibaryon field, two insertions of $V_{\pi}$, and more counterterms. The phase-shifts at various orders in $V_{\pi}$ are shown in fig. 16.

\section{References}

[1] S. R. Beane, P. F. Bedaque, W. C. Haxton, D. R. Phillips and M. J. Savage, Essay for the Festschrift in honor of Boris Ioffe, to appear in the 'Encyclopedia of Analytic QCD', edited by M. Shifman, to be published by World Scientific. hep-ph/0008064.

[2] U. van Kolck, Prog. Part. Nucl. Phys. 43, 337 (1999).

[3] T. -S. Park, K. Kubodera, D. -P. Min and M. Rho, Nucl. Phys. A646, 83 (1999).

[4] E. Epelbaum, H. Kamada, A. Nogga, H. Witala, W. Glöckle and U.-G. Meißner, nucl-th/0009067.

[5] Nuclear Physics with Effective Field Theory, and INT workshop, (1999), edited by P. F. Bedaque, M. J. Savage, R. Seki and U. van Kolck, World Scientific (2000). ISBN 981-02-4181-X.

[6] S. Weinberg, Phys. Lett. B251, 288 (1990); Nucl. Phys. B363, 3 (1991); Phys. Lett. B295, 114 (1992).

[7] M. Lutz, hep-ph/9606301 p. 22 (1996); U. van Kolck, in Mainz 1997, Chiral Dynamics: Theory and Experiment, ed. A. M. Bernstein, D. Drechsel, and T. Walcher, (Springer-Verlag, Berlin, 1998); D. B. Kaplan, M. J. Savage, and M. B. Wise, Nucl. Phys. B534, 329 (1998); D. B. Kaplan, M. J. Savage, and M. B. Wise, Phys. Lett. B424, 390 (1998); U. van Kolck, Nucl. Phys. A645, 273 (1999); J.-W. Chen, G. Rupak, and M. J. Savage, Nucl. Phys. A653, 386 (1999). 
[8] D. B. Kaplan, M. J. Savage, and M. B. Wise, Nucl. Phys. B478 629 (1996).

[9] D. B. Kaplan, M. J. Savage, and M. B. Wise, Phys. Lett. B424, 390 (1998); Nucl. Phys. B534, 329 (1998).

[10] S. Fleming T. Mehen and I. W. Stewart, Nucl.Phys. A677, 313 (2000); Phys. Rev. C61, 044005 (2000).

[11] C. Ordonez, L. Ray and U. van Kolck, phys. Rev. C53, 2086 (1996).

[12] E. Epelbaum, W. Glockle and U. Meissner, Nucl. Phys. A671, 295 (2000).

[13] A. M. Perelomov and V. S. Popov, Teor. i. Mate. Fiz. 4, 48 (1970).

[14] W. M. Frank, D. J. Land, and R. M. Spector, Rev. Mod. Phys. 43, 36 (1971).

[15] S. R. Beane, P. F. Bedaque, L. Childress, A. Kryjevski, J. McGuire and U. van Kolck, quant-ph/0010073.

[16] D. B. Kaplan and J. V. Steele, Phys. Rev. C60, 064002 (1999).

[17] V. G. J. Stoks, R. A. M. Klomp, M. C. M. Rentmeester, and J. J. de Swart, Phys. Rev. C48, 792 (1993).

[18] D. B. Kaplan, Nucl. Phys. B494, 471 (1997).

[19] S. R. Beane and M. J. Savage, nucl-th/0011067.

[20] D. R. Phillips, G. Rupak, and M. J. Savage, Phys. Lett. B473, 209 (2000).

[21] K. A. Scaldeferri, D. R. Phillips, C. W. Kao, T. D. Cohen Phys. Rev. C56, 679 (1997).

[22] G. P. Lepage, hucl-th/9706029.

[23] P. F. Bedaque and H. W. Grießhammer, Nucl. Phys. A671, 357 (2000).

[24] K. G. Richardson, M. C. Birse and J. A. McGovern, hep-ph/9708435, Phys. Lett. B464, 169 (1999); 9808398; PiN Newslett. 15, 280 (1999).

[25] P.F. Bedaque, H.-W. Hammer, and U. van Kolck, Nucl. Phys. A646, 444 (1999); P.F. Bedaque, H.-W. Hammer, and U. van Kolck, Phys. Rev. Lett. 82, 463 (1999); H.W. Hammer and T. Mehen, nucl-th/0011024.

[26] T. Frederico, V. S. Timoteo, and L. Tomio, Nucl. Phys. A653, 209 (1999).

[27] D. R. Phillips and T. D. Cohen, Nucl. Phys. A668, 45 (2000).

[28] D. W. L. Sprung, W. van Dijk, E. Wang, D. C. Zheng, P. Sarriguren and J. Martorell, Phys. Rev. C49, 2942 (1994). 
[29] S. R. Sharpe and N. Shoresh, hep-lat/0011089; Phys. Rev. D62, 094503 (2000); Nucl. Phys. Proc. Suppl. 83, 968 (2000); S. R. Sharpe, Phys. Rev. D56, 7052 (1997); C. W. Bernard and M. F. L. Golterman, Phys. Rev. D49, 486 (1994).

[30] J. Gasser and H. Leutwyler, Nucl. Phys. B250, 465 (1985).

[31] R. Wiringa, Private Communication.

[32] A. Bulgac, G. A. Miller and M. Strikman, Phys. Rev. C56, 3307 (1997).

[33] J. Gasser and H. Leutwyler, Annals Phys. 158, 142 (1984).

[34] G. Colangelo, J. Gasser and H. Leutwyler, hep-ph/0103088.

[35] U.-G. Meißner, Essay for the Festschrift in honor of Boris Ioffe, to appear in the 'Encyclopedia of Analytic QCD', edited by M. Shifman, to be published by World Scientific. hep-ph/0007092.

[36] N. Fettes and U.-G. Meißner, Nucl. Phys. A676, 311 (2000).

[37] M. Rho, contribution to the proceedings of the INT workshop on Nuclear Physics with Effective Field Theory II, edited by P.F. Bedaque, M. J. Savage, R. Seki and U. van Kolck, World Scientific (2000). ISBN 981-02-4181-X.

[38] M. Fukugita, Y. Kuramashi, M. Okawa, H. Mino and A. Ukawa, Phys. Rev. D52, 3003 (1995).

[39] M. Lüscher, Commun. Math. Phys. 105, 153 (1986).

[40] D. B. Kaplan, M. J. Savage and M. B. Wise, Phys. Rev. C59, 617 (1999).

[41] W. C. Haxton and C. L. Song, hucl-th/9906082, Phys. Rev. Lett. 84, 5454 (2000);

W. C. Haxton and T. Luu, nucl-th/0101022.

[42] G. Rupak and N. Shoresh, Phys. Rev. C60, 054004 (1999). 